\title{
Least-looping stepping-stone-based ASM approach for transportation and triangular intuitionistic fuzzy transportation problems
}

\author{
Kedar Nath Das ${ }^{1} \cdot$ Rajeev Das ${ }^{1}$ - Debi Prasanna Acharjya ${ }^{2}$
}

Received: 2 December 2020 / Accepted: 10 July 2021 / Published online: 26 July 2021

(c) The Author(s) 2021

\begin{abstract}
Transportation problem (TP) is a popular branch of Linear Programming Problem in the field of Transportation engineering. Over the years, attempts have been made in finding improved approaches to solve the TPs. Recently, in Quddoos et al. (Int J Comput Sci Eng (IJCSE) 4(7): 1271-1274, 2012), an efficient approach, namely ASM, is proposed for solving crisp TPs. However, it is found that ASM fails to provide better optimal solution in some cases. Therefore, a new and efficient ASM appoach is proposed in this paper to enhance the inherent mechanism of the existing ASM method to solve both crisp TPs and Triangular Intuitionistic Fuzzy Transportation Problems (TIFTPs). A least-looping stepping-stone method has been employed as one of the key factors to improve the solution quality, which is an improved version of the existing steppingstone method (Roy and Hossain in, Operation research Titus Publication, 2015). Unlike stepping stone method, least-looping stepping-stone method only deals with few selected non-basic cells under some prescribed conditions and hence minimizes the computational burden. Therefore, the framework of the proposed method (namely LS-ASM) is a combination of ASM (Quddoos et al. 2012) and least-looping stepping-stone approach. To validate the performance of LS-ASM, a set of six case studies and a real-world problem (those include both crisp TPs and TIFTPs) have been solved. The statistical results obtained by LS-ASM have been well compared with the existing popular modified distribution (MODI) method and the original ASM method, as well. The statistical results confirm the superiority of the LS-ASM over other compared algorithms with a less computationl effort.
\end{abstract}

Keywords Transportation problem · Triangular intuitionistic fuzzy number · Stepping-stone method · ASM method · Optimal solution

\section{Introduction}

A constrained optimization problem consists of an objective function along with some constraints. If all of them are linear, then it is known as a Linear Programming Problem (LPP). The popular Transportation Problem (TP) is a particular case of LPP. The objective is to minimize the cost

Rajeev Das

rajeev_rs@math.nits.ac.in

Kedar Nath Das

kedar.iitr@gmail.com

Debi Prasanna Acharjya

dpacharjya@gmail.com

1 Department of Mathematics, National Institute of Technology Silchar, Silchar, India

2 School of Computer Science and Engineering, VIT-Vellore, Vellore, India of dispensing products from ' $m$ ' number of suppliers to ' $n$ ' number of different stations. The LPP formulation of the TP is originally developed by Hitchcock [1] in the year 1941, as follows.

\section{Mathematical formulation of TP [1]}

Consider a TP with ' $m$ ' sources and ' $n$ ' destinations, where $p_{i j}$ is the unit transportation cost from $i$ th source to $j$ th destination. Let $S_{i}$ be the supply quantity available at $i$ th source and $D_{j}$ be the demand quantity of $j$ th destination. The goal of the objective function is to minimize the total transportation cost $(Z)$, subject to the commodity $q_{i j}$ is shipped that met the demand. The mathematical formulation of a balanced TP (where $\sum_{i=1}^{m} S_{i}=\sum_{j=1}^{n} D_{i}$ ) is given by 
Minimize $Z=\sum_{i=1}^{m} \sum_{j=1}^{n} p_{i j} q_{i j}$

subject to

$\sum_{j=1}^{n} q_{i j} \leq S_{i} ; i=1,2, \ldots, m$

$\sum_{i=1}^{m} q_{i j} \geq D_{j} ; j=1,2, \ldots, n$

$q_{i j} \geq 0 \forall i, j$.

The first two set of constraints fortify that the total quantities shipped from the source ' $i$ ' must not exceed its supply and the total quantity shipped to the ' $j$ th' destination must be greater than or equal to its demand, respectively. The third set of constraints assures that quantity of commodity $q_{i j}$ must be non-negative.

Real-world distribution problems are uncertain in nature because of the changes taking place in the parameters. Zadeh [2] developed the fuzzy set theory to deal with indistinct data computationally in making decisions and succeeded in its applications in different areas. The application of fuzzy set theory landed in the optimization field after the spearheading research carried out by Bellman and Zadeh [3]. Nonetheless, there are uncertainties with hesitation due to numerous factors like need for better communication, inaccuracy in data, comprehension of markets, unawareness of customers, etc. Likewise, the costs are still hanging with uncertainties with hesitation because of different components like diversity in rates of fuels, congested driving conditions, climatic conditions, and so on. In such circumstances, the decision-makers fail to foresee transportation cost precisely. Therefore, to deal with these uncertain data, Atanassov [4] proposed the Intuitionistic Fuzzy Set (IFS) which is validated to be more accurate than the fuzzy set theory proposed by Zadeh [2]. Afterward, quite ample number of successful approaches have been presented in the literature to solve TPs and IFTPs and the range of application has been steadily widened. It is now considered as one of the important tools in business and industry. Several sorts of methods have been proposed for finding the optimal solution. ASM [5] is one of such recent methods which has been applied to solve TPs and IFTPs, as well. The authors of ASM method solved a real-valued TP and then compared its result to that of MODI method. They concluded ASM to be a quick and effective approach. In the year 2012, Mohammad Kamrul Hasan [15] proposed that direct methods (including ASM method) for finding optimal solution of a TP do not reflect optimal solution continuously. Murugesan [17] admitted and perceived Hasan's claim by verifying the ASM method for several benchmark problems. However, in the mean time, Abdul Quddoos et al. [16] experienced a few problems for which the ASM method does not happen to provide the optimal solution, but executes well enough to provide a best IBFS, which appears to be close to the optimal solution. To resolve this issue, Abdul Quddoos et al. [16] forwarded a revised approach to the ASM method where MODI method was combined with the existing ASM method. This approach gives the optimal solution directly for most of the problems, and if not, it gives the best IBFS. Later, the claim was established by Murugesan et al. [18-23] in which they tested on 30 balanced and 20 unbalanced benchmark TPs. Over the years, it can be seen that several approaches have been mentioned in the literature for the improvement of ASM method. However, the concept of stepping stone was never used with ASM method in the past research. Therefore, an effort is made in this manuscript to improve the efficiency of the original ASM by a crucial modification in it. The authors developed a new approach, namely, the least-looping stepping-stone approach, and hybridized it with ASM to increase the robustness of the original ASM.

The manuscript is systemized as follows: Section "Motivation" presents the motivation of the proposed method; Section "Proposed LS-ASM Method" introduces the proposed algorithm alongwith six case studies and a real-life problem. Section Results and Discussion includes the comparative analysis of results. The conclusion part of this paper is produced in Section Conclusion, which comprises some of the future opportunities, as well.

\section{Motivation}

To solve TPs, a number of suitable approaches have been presented in the literature. Most of these approaches attempted to calculate the Initial Basic Feasible Solution (IBFS) before employing its different steps. In 2012, a popular method, namely ASM, was proposed [5] to solve the classical TP without finding the IBFS. In fact, it was a direct approach basically named after the first letters of the authors' names Abdul Quddoos, Shakeel Javaid, and M.M. Khalid. However, later in $[8,11]$, it is seen that ASM failed to get to the optimal cost for some of the TPs. To validate this fact, two case studies of Crisp TPs are explained below.

\section{Case study-1:}

Table 1 considers a balanced TP which is solved by ASM Method in Table 2 with total transportation cost $=\mathbf{7} \times \mathbf{5}+\mathbf{5}$ $\times 5+9 \times 20+3 \times 25+3 \times 20+2 \times 5+3 \times 10=415$.

\section{Case study-2:}

Table 3 considers a balanced TP which is solved by ASM method in Table 4 with total transportation cost $=\mathbf{1 3} \times \mathbf{8 0}$ $+15 \times 200+22 \times 50+11 \times 280+19 \times 220+11 \times 180=1$ 4,380 . 
Table 1 A balanced minimization TP [8]

\begin{tabular}{llllll}
\hline & $D_{1}$ & $D_{2}$ & $D_{3}$ & $D_{4}$ & Supply \\
\hline$S_{1}$ & 7 & 5 & 9 & 11 & 30 \\
$S_{2}$ & 4 & 3 & 8 & 6 & 25 \\
$S_{3}$ & 3 & 8 & 10 & 5 & 20 \\
$S_{4}$ & 2 & 6 & 7 & 3 & 15 \\
Demand & 30 & 30 & 20 & 10 & 90 \\
\hline
\end{tabular}

Table 2 Optimal table according to ASM [5]

\begin{tabular}{llllll}
\hline & $D_{1}$ & $D_{2}$ & $D_{3}$ & $D_{4}$ & Supply \\
\hline$S_{1}$ & $7_{5}$ & $5_{5}$ & $9_{20}$ & 11 & 30 \\
$S_{2}$ & 4 & $3_{25}$ & 8 & 6 & 25 \\
$S_{3}$ & $3_{20}$ & 8 & 10 & 5 & 20 \\
$S_{4}$ & $2_{5}$ & 6 & 7 & $3_{10}$ & 15 \\
Demand & 30 & 30 & 20 & 10 & 90 \\
\hline
\end{tabular}

Bold values in the respective mentioned tables signify equally optimal or better optimal solutions

Table 3 A balanced minimization TP [11]

\begin{tabular}{llllll}
\hline & $D_{1}$ & $D_{2}$ & $D_{3}$ & $D_{4}$ & Supply \\
\hline$S_{1}$ & 13 & 15 & 16 & 18 & 280 \\
$S_{2}$ & 20 & 22 & 11 & 8 & 330 \\
$S_{3}$ & 19 & 25 & 17 & 11 & 400 \\
Demand & 300 & 250 & 280 & 180 & 1010 \\
\hline
\end{tabular}

Table 4 Optimal table according to ASM [5]

\begin{tabular}{llllll}
\hline & $D_{1}$ & $D_{2}$ & $D_{3}$ & $D_{4}$ & Supply \\
\hline$S_{1}$ & $13_{80}$ & $15_{200}$ & 16 & 18 & 280 \\
$S_{2}$ & 20 & $22_{50}$ & $11_{280}$ & 8 & 330 \\
$S_{3}$ & $19_{220}$ & 25 & 17 & $11_{180}$ & 400 \\
Demand & 300 & 250 & 280 & 180 & 1010 \\
\hline
\end{tabular}

Bold values in the respective mentioned tables signify equally optimal or better optimal solutions

It is worth noting that using each of the techniques like Vogel's Approximation Method (VAM)-MODI [9], NorthWest Corner Method (NWCM)-MODI [9], Row Minima method (RMM)-MODI [9], and Column Minima Method (CMM)-MODI [9], the optimal solutions found are $\mathbf{4 1 0}$ and $\mathbf{1 4 , 1 8 0}$ for case studies- 1 and 2 , respectively. It motivated the authors to investigate more key factors of ASM, which needed to be substantially modified to enhance its robustness. Least-looping stepping-stone approach has been employed in the working mechanism of ASM to improve the robustness of ASM. This method is exempted from checking the optimality criteria for all the non-basic cells. Instead, it checks for some selected loops and calculates the net cost change values. Therefore, it helps to minimize the computational time as compared to stepping-stone approach. This new approach is proven to be more attactive due to easy implementation and minimum calculation, as compared to the classical approaches. To validate the better performance of the proposed method in all sort of TPs, two problems on balanced TPs, two problems on TIFTPs, two problems on unbalanced TPs, and one real-life problem have been considered. The working mechanism of the proposed approach has been explained in details in the following section.

\section{Proposed LS-ASM Method}

\section{Least-looping stepping-stone method}

The stepping-stone Method [14] emerged from the idea of crossing a pond making use of stepping stones. The entire cost matrix is considered as a pond and the basic cells are the stones required for certain sequential movement within the pond. In other words, it is an iterative approach to reach the optimal solution from an IBFS. Each iteration includes the formation of closed loops* for each of the non-basic cells in the cost matrix. This helps in determining whether the current iteration has all the best routes. If not, then the only penalty imposed is to perform additional iterations.

However in this paper, the stepping-stone Method is suitably modified for its up gradation. Unlike ASM, LS-ASM considers only few selected non-basic cells for forming closed loops in its working mechanism (algorithm) presented below. First two iterations of least-looping steppingstone are combined with ASM to reach the optimal solution. This new method is named as 'Least-looping stepping-stone based ASM Method' (LS-ASM Method). Even after this hybridization, the proposed method proves out to be an easy and time-saving approach. In the later part of this paper, LSASM has been compared with other mentioned approaches.

\section{LS-ASM algorithm}

Consider the given TP and construct its transportation table.

Step1: If the transportation table is not balanced, then balance it by adding suitable dummy rows/columns. Else, proceed.

Step 2: Subtract each row minima from the corresponding row costs and each column minima from the corresponding column costs.

Step 3: Select a zero appearing in the cost matrix and count the total sum of the number of zeros (precluding the selected zero) in that corresponding row and column. Let us name it as sum-zero. Find the sum-zero for each of the existing zeros in the cost matrix.

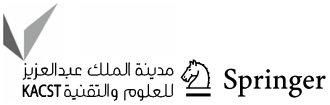


Step 4: Choose a cell with the cost zero for which the sumzero is minimum. Supply maximum possible unit to that zero cell. If tie occurs for some zeros in step 3, then take that zero cell for which total sum of all the costs of respective row and column is maximum and assign maximum possible unit to that cell.

Step 5: Block the row/column where the supply or the demand is met.

Step 6: Now, if the reduced cost matrix does not have at least one zero in each row and each column, then go to step 2; otherwise continue step 3 to step 5 until all the demands and supplies are met.

Step 7: Write the resulting table with both basic and nonbasic cells.

Step 8: For each non-basic cell in the resulting table, trace closed loops* back to the original non-basic cell. Choose only those closed loops* where either or both costs of the adjacent two basic cells of a non-basic cell are greater than the costs of the corresponding non-basic cells.

Step 9: For each selected closed loop* by Step 8, assign $(+)$ and (-) sign alternatively on each corner, starting from the original non-basic cell. Choose the minimum allocated value of all the negative positions (-) on the closed loop*. Allocate this value to the selected nonbasic cell, so that the non-basic cell becomes basic cell. Add this value to other basic cells with (+) sign and subtract this value from other basic cells with (-) sign.

Step 10: Calculate the sum of the transportation costs of each cell followed in the closed loop*. This sum value is termed as Net Cost Change. Redo this for all other selected closed loops*.

Step 11: If all the net cost change values are non-negative, an optimal value is attained. Then terminate. Else, select the non-basic cell having negative net cost change value and draw a closed loop.

Step 12: Choose the minimum allocated value of all the negative positions (-) on the closed loop. Allocate this value to the selected non-basic cell, so that the non-basic cell becomes basic cell. Add this value to other basic cells with $(+)$ sign and subtract this value from other basic cells with (-) sign.

Step 13: Repeat the steps 8-11 (viz., steps of Least-looping stepping-stone Method) once again and the optimal solution will be obtained with all the values of net cost change non-negative for non-basic cells.

Step 14: Evaluate the transportation cost and stop.

* Closed loop is a sequence of cells that forms a look in the transportation table with the following conditions.

1. Any two consecutive cells lie either in the similar row/ column.

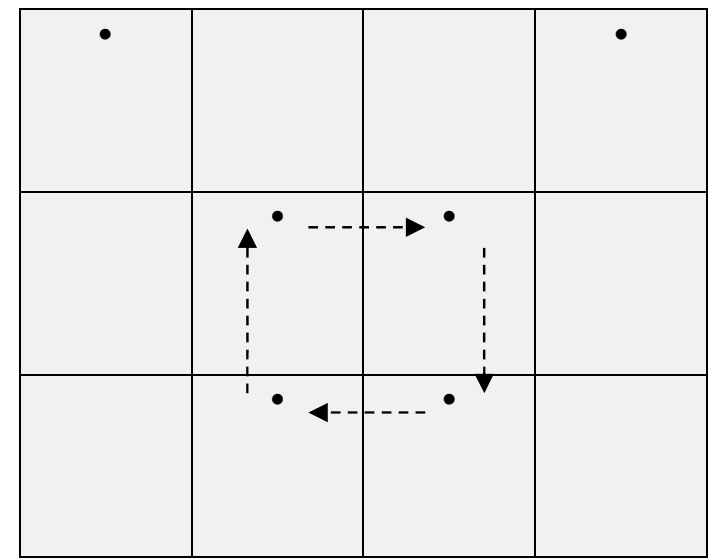

Fig. 1 One form of closed loop*

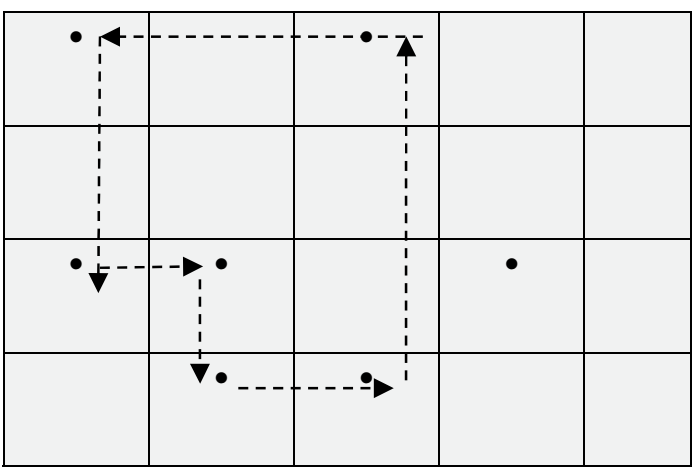

Fig. 2 Another form of closed loop*

2. No three successive cells lie either in the similar row/ column.

3. The starting and ending cell of a sequence lies in the similar row/column.

4. No cell shows up more than once in a sequence.

5. Only horizontal and vertical moves are permitted and can change directions at used cells.

To have a quick reference of closed loops* in LS-ASM, two illustrations are presented in Figs. 1 and 2.

To have a quick reference of the work sequence in LSASM, the flow diagram also is presented in Fig. 3.

\section{Case studies}

In this section, an attempt is made to justify the efficiency of the proposed LS-ASM method in solving TPs. Hence, a set of varieties of TPs are being considered both from crisp and fuzzy environment. Keeping in view the above fact, the TPs of the following categories have been solved by LS-ASM. 


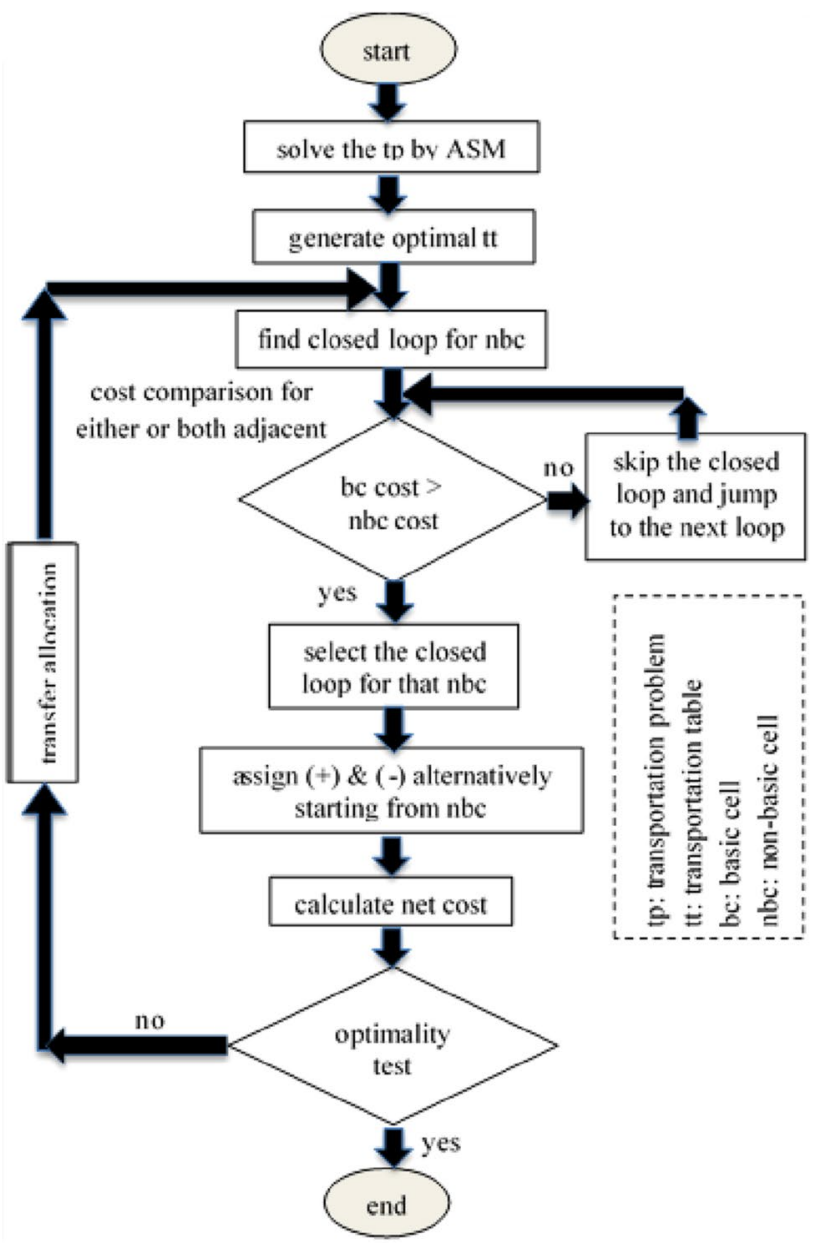

Fig. 3 Flowchart for LS-ASM

- Balanced TPs in crisp environment (case studies-1 and 2)

- Balanced TPs in intuitionistic fuzzy environment (case studies-3 and 4)

- Unbalanced TPs in crisp environment (case studies-5 and 6)

- A real-life problem in intuitionistic fuzzy environment (separately discussed in Section "Real-Life IFTP").

Now, the detailed descriptions of the above TPs are dicussed below under individual case studies.

\section{Balanced TPs}

\section{Case study-1:}

The first case study is already considered in Sect. 2 . Assuming the optimal table by ASM, clearly the non-basic cells are $S_{1} D_{4}, S_{2} D_{1}, S_{2} D_{3}, S_{2} D_{4}, S_{3} D_{2}, S_{3} D_{3}, S_{3} D_{4}, S_{4} D_{2}$, and $S_{4} D_{3}$.

In Table 5, it is observed from the loop that $S_{1} D_{1}$ and $S_{4} D_{4}$ are adjacent to $S_{1} D_{4}$. Now, the cost values of $S_{1} D_{1}$ and $S_{4} D_{4}$ (7 and 3, respectively) are less than the cost value of $S_{1} D_{4}$
Table 5 Closed loop for the non-basic cell $S_{1} D_{4}$

\begin{tabular}{|c|c|c|c|c|c|}
\hline & $D_{1}$ & $D_{2}$ & $D_{3}$ & $D_{4}$ & Supply \\
\hline$S_{1}$ & $(-) 75$ & 55 & 920 & $f^{(+) 11}$ & 30 \\
\hline$S_{2}$ & 4 & 325 & 8 & 6 & 25 \\
\hline$S_{3}$ & 320 & 8 & 10 & 5 & 20 \\
\hline$S_{4}$ & $(+) 2_{5}$ & 6 & 7 & $-(-) 3 \quad 10$ & 15 \\
\hline Demand & 30 & 30 & 20 & 10 & 90 \\
\hline
\end{tabular}

Bold values in the respective mentioned tables signify equally optimal or better optimal solutions

(i.e., 11). Therefore, this closed loop is not considered to evaluate the net cost change value. Other closed loops can be constructed and checked in the similar way for the other non-basic cells.

Now, apply Step 12 of the LS-ASM algorithm to the non-basic cell $S_{2} D_{1}$ having the only negative net cost change value (Refer to Table 6). It is seen that in the closed loop of Table 7 out of both the negative positions, $5<25$. Therefore, 5 is added to the non-basic cell $S_{2} D_{1}$ and basic cell $S_{1} D_{2}$, whereas it is subtracted from the allocated values of the basic cells $S_{1} D_{1}$ and $S_{2} D_{2}$. Now, $S_{2} D_{1}$ becomes a basic cell. Since, it can be checked that all the net cost change values of the non-basic cells are positive in the next iteration. Finally, the optimal solution has been reached. The optimal table of the TP is demonstrated in Table 8.

Thus, the total transportation cost according to LSASM Method $=5 \times \mathbf{1 0}+\mathbf{9} \times \mathbf{2 0}+\mathbf{4} \times \mathbf{5}+\mathbf{3} \times \mathbf{2 0}+\mathbf{3} \times \mathbf{2 0}$ $+2 \times 5+3 \times 10=410$.

\section{Case study-2:}

The case study-2 has already been considered in Sect. 2 . Proceeding in the similar way as done for case study-1, the optimal transportation cost by LS-ASM is obtained as $\mathbf{1 4 , 1 8 0}$. Two more case studies with the implementation of LS-ASM for TIFTPs are described following case studies.

\section{Case study-3:}

Table 10 uses the ranking function [6] to convert the Triangular Intuitionistic Fuzzy cost values to real values. In Table 11, the total transportation cost according to the LS-ASM Method $=1 \times 4+4 \times 1+7 \times 5+8 \times 1+2 \times 7+$ $6 \times 3+5 \times 7=118$.

\section{Case study-4:}


Table 6 Selected closed loops for each of the non-basic cells

\begin{tabular}{|c|c|c|}
\hline Non-basic cells & Closed loops & Net cost change \\
\hline$S_{2} D_{1}$ & $(+) S_{2} D_{1}(+) S_{2} D_{2^{--}}+(+) S_{1} D_{2^{--(-)}} S_{1} D_{1-(+)} S_{2} D_{1}$ & $4-3+5-7=-1$ (negative) \\
\hline$S_{2} D_{3}$ & $(+) S_{2} D_{3}(-) S_{1} D_{3}-S_{1} D_{2--(-)} S_{2} D_{2}-(+) S_{2} D_{3}$ & $8-9+5-3=1$ \\
\hline$S_{4} D_{2}$ & $(+) S_{4} D_{2}(-) S_{4} D_{1^{--}}+(+) S_{1} D_{1^{--}-(-) S_{1} D_{2}-(+) S_{4} D_{2}}$ & $6-2+7-5=6$ \\
\hline$S_{4} D_{3}$ & $(+) S_{4} D_{3}(-) S_{4} D_{1^{--}}+(+) S_{1} D_{1^{--}-(-) S_{1} D_{3}-(+) S_{4} D_{3}}$ & $7-2+7-9=3$ \\
\hline
\end{tabular}

Table 7 Closed loop for the non-basic cell $S_{2} D_{1}$

\begin{tabular}{|c|c|c|c|c|c|}
\hline & $D_{1}$ & $D_{2}$ & $D_{3}$ & $D_{4}$ & Supply \\
\hline$S_{1}$ & $(-) 7_{5}$ & $-(+) 5_{5}$ & 920 & 11 & 30 \\
\hline$S_{2}$ & $(+) 4$ & $(-) 3_{25}$ & 8 & 6 & 25 \\
\hline$S_{3}$ & 320 & 8 & 10 & 5 & 20 \\
\hline$S_{4}$ & 25 & 6 & 7 & 310 & 15 \\
\hline Demand & 30 & 30 & 20 & 10 & 90 \\
\hline
\end{tabular}

Bold values in the respective mentioned tables signify equally optimal or better optimal solutions

Table 8 Optimal result table

\begin{tabular}{llllll}
\hline & $D_{1}$ & $D_{2}$ & $D_{3}$ & $D_{4}$ & Supply \\
\hline$S_{1}$ & 7 & $5_{10}$ & $9_{20}$ & 11 & 30 \\
$S_{2}$ & $4_{5}$ & $3_{20}$ & 8 & 6 & 25 \\
$S_{3}$ & $3_{20}$ & 8 & 10 & 5 & 20 \\
$S_{4}$ & $2_{5}$ & 6 & 7 & $3_{10}$ & 15 \\
Demand & 30 & 30 & 20 & 10 & 90 \\
\hline
\end{tabular}

Bold values in the respective mentioned tables signify equally optimal or better optimal solutions

Table 13 uses the ranking function [6] to convert the Triangular Intuitionistic Fuzzy cost values to real values. In Table 14, the total transportation cost according to the LS-ASM Method $=(243.33 \times 3500+3721.21 \times 1000+4$ $06.833 \times 1500+1050 \times 2000+2209.259 \times 1500+5621$. $21 \times 500)=13,406,940.5$.
Table 10 Transportation of Table 9 after using the ranking function [6]

\begin{tabular}{llllll}
\hline & $D_{1}$ & $D_{2}$ & $D_{3}$ & $D_{4}$ & Supply \\
\hline$S_{1}$ & 16 & 1 & 8 & 13 & 4 \\
$S_{2}$ & 11 & 4 & 7 & 10 & 6 \\
$S_{3}$ & 8 & 15 & 9 & 2 & 8 \\
$S_{4}$ & 6 & 12 & 5 & 14 & 10 \\
Demand & 4 & 5 & 12 & 7 & 28 \\
\hline
\end{tabular}

Table 11 Optimal solution table using LS-ASM

\begin{tabular}{llllll}
\hline & $D_{1}$ & $D_{2}$ & $D_{3}$ & $D_{4}$ & Supply \\
\hline$S_{1}$ & 16 & $1_{4}$ & 8 & 13 & 4 \\
$S_{2}$ & 11 & $4_{1}$ & $7_{5}$ & 10 & 6 \\
$S_{3}$ & $8_{1}$ & 15 & 9 & $2_{7}$ & 8 \\
$S_{4}$ & $6_{3}$ & 12 & $5_{7}$ & 14 & 10 \\
Demand & 4 & 5 & 12 & 7 & 28 \\
\hline
\end{tabular}

Bold values in the respective mentioned tables signify equally optimal or better optimal solutions

\section{Unbalanced TPs}

In this sub-section, two unbalanced TPs are considered as follows to solve by LS-ASM Method.

\section{Case study-5:}

Table 15 considers an unbalanced TP which is solved by LS-ASM in Table 16 with total transportation cost $=\mathbf{2 5} \times$ $125+15 \times 450+0 \times 300+10 \times 350+20 \times 225=17,875$.

Table 9 A balanced minimization TP with TIFNs [10]

\begin{tabular}{llllll}
\hline & $D_{1}$ & $D_{2}$ & $D_{3}$ & $D_{4}$ & IF supply \\
\hline$S_{1}$ & $(14,16,18 ; 12,16,20)$ & $(0,1,2 ;-1,1,3)$ & $(7,8,9 ; 6,8,10)$ & $(11,13,15 ; 10,13,16)$ & $(2,4,6 ; 1,4,7)$ \\
$S_{2}$ & $(8,11,14 ; 7,11,15)$ & $(3,4,5 ; 2,4,6)$ & $(5,7,9 ; 4,7,10)$ & $(8,10,12 ; 6,10,14)$ & $(5,6,7 ; 4,6,8)$ \\
$S_{3}$ & $(6,8,10 ; 5,8,11)$ & $(13,15,17 ; 12,15,18)$ & $(7,9,11 ; 6,9,12)$ & $(1,2,3 ; 0,2,4)$ & $(7,8,9 ; 5,8,11)$ \\
$S_{4}$ & $(5,6,7 ; 4,6,8)$ & $(11,12,13 ; 10,12,14)$ & $(3,5,7 ; 1,5,9)$ & $(12,14,16 ; 11,14,17)$ & $(8,10,12 ; 6,10,14)$ \\
IF Demand & $(3,4,5 ; 2,4,6)$ & $(3,5,7 ; 1,5,9)$ & $(10,12,14 ; 8,12,16)$ & $(6,7,8 ; 5,7,9)$ & \\
\hline
\end{tabular}


Table 12 A balanced minimization TP with TIFNs [7]

\begin{tabular}{|c|c|c|c|c|c|}
\hline & $D_{1}$ & $D_{2}$ & $D_{3}$ & $D_{4}$ & $\begin{array}{l}\text { Supply } \\
\text { (in tons) }\end{array}$ \\
\hline$S_{1}$ & $\begin{array}{l}(210,250,270 ; 200,250, \\
280)\end{array}$ & $\begin{array}{l}(600,700,750 ; 600,700, \\
800)\end{array}$ & $\begin{array}{l}(950,1000,1050 ; 900,1000, \\
1100)\end{array}$ & $\begin{array}{l}(3500,3700,3900 ; 3400, \\
3700,4100)\end{array}$ & 4500 \\
\hline$S_{2}$ & $\begin{array}{l}(650,750,800 ; 600,750, \\
850)\end{array}$ & $\begin{array}{l}(350,400,450 ; 340,400, \\
\quad 480)\end{array}$ & $\begin{array}{l}(1000,1050,1100 ; 950, \\
1050,1150)\end{array}$ & $\begin{array}{l}(3600,3900,4000 ; 3500, \\
3900,4600)\end{array}$ & 3500 \\
\hline$S_{3}$ & $\begin{array}{l}(2600,2800,3000 ; 2500, \\
2800,3100)\end{array}$ & $\begin{array}{l}(2100,2200,2300 ; 2100, \\
2200,2350)\end{array}$ & $\begin{array}{l}(2900,3100,3300 ; 2800, \\
3100,3400)\end{array}$ & $\begin{array}{l}(5400,5600,5800 ; 5300, \\
5600,6000)\end{array}$ & 2000 \\
\hline $\begin{array}{l}\text { Demand } \\
\text { (in } \\
\text { tons) }\end{array}$ & 3500 & 3000 & 2000 & 1500 & 10,000 \\
\hline
\end{tabular}

Table 13 Transportation of Table 12 after using the ranking function [6]

\begin{tabular}{llllll}
\hline & $D_{1}$ & $D_{2}$ & $D_{3}$ & $D_{4}$ & $\begin{array}{l}\text { Supply } \\
\text { (in tons) }\end{array}$ \\
\hline$S_{1}$ & 243.33 & 692.857 & 1000 & 3721.21 & 4500 \\
$S_{2}$ & 733.33 & 406.388 & 1050 & 4015.87 & 3500 \\
$S_{3}$ & 2800 & 2209.259 & 3100 & 5621.21 & 2000 \\
$\begin{array}{l}\text { Demand } \\
\text { (in tons) }\end{array}$ & 3500 & 3000 & 2000 & 1500 & 10,000 \\
\hline
\end{tabular}

Table 14 Optimal solution table using LS-ASM

\begin{tabular}{llllll}
\hline & $D_{1}$ & $D_{2}$ & $D_{3}$ & $D_{4}$ & $\begin{array}{l}\text { Supply } \\
\text { (in tons) }\end{array}$ \\
\hline$S_{1}$ & $243.33_{3500}$ & 692.857 & 1000 & $3721.21_{1000}$ & 4500 \\
$S_{2}$ & 733.33 & $406.388_{1500}$ & $1050_{2000}$ & 4015.87 & 3500 \\
$S_{3}$ & 2800 & $2209.259_{1500}$ & 3100 & $5621.21_{500}$ & 2000 \\
$\begin{array}{l}\text { Demand } \\
\quad \text { (in }\end{array}$ & 3500 & 3000 & 2000 & 1500 & 10,000 \\
tons) & & & & & \\
\hline
\end{tabular}

Bold values in the respective mentioned tables signify equally optimal or better optimal solutions

Table 15 An unbalanced minimization TP [12]

\begin{tabular}{|c|c|c|c|c|}
\hline \multirow{2}{*}{$\begin{array}{c}\text { From/ To } \\
\text { Plants }\end{array}$} & \multicolumn{2}{|c|}{ Warehouse(j) } & \multirow{2}{*}{ Capacity } \\
\cline { 2 - 5 } & $\mathrm{P}$ & $\mathrm{Q}$ & $\mathrm{R}$ & \\
\hline A & 35 & 25 & 15 & 875 \\
\hline B & 10 & 20 & 30 & 575 \\
\hline Demand & 350 & 350 & 450 & 1450 \\
\hline
\end{tabular}

Table 16 Optimal solution table using LS-ASM

\begin{tabular}{|c|c|c|c|c|c|}
\hline \multirow{2}{*}{$\begin{array}{c}\text { From/ To } \\
\text { Plants }\end{array}$} & \multicolumn{4}{|c|}{ Warehouse(j) } & \multirow{2}{*}{ Capacity } \\
\cline { 2 - 6 } & $\mathrm{P}$ & $\mathrm{Q}$ & $\mathrm{R}$ & Dummy & \\
\hline $\mathrm{A}$ & 35 & $25_{\mathbf{1 2 5}}$ & $15_{\mathbf{4 5 0}}$ & $0_{\mathbf{3 0 0}}$ & 875 \\
\hline B & $10_{\mathbf{3 5 0}}$ & $20_{\mathbf{2 2 5}}$ & 30 & 0 & 575 \\
\hline Demand & 350 & 350 & 450 & 300 & 1450 \\
\hline
\end{tabular}

Bold values in the respective mentioned tables signify equally optimal or better optimal solutions

Table 17 An unbalanced minimization TP [13]

\begin{tabular}{|c|c|c|c|c|}
\hline$S_{s_{i}} d_{j}$ & $d_{1}$ & $d_{2}$ & $d_{3}$ & Supply \\
\hline$s_{1}$ & 5 & 1 & 7 & 10 \\
\hline$s_{2}$ & 6 & 4 & 6 & 80 \\
\hline$s_{3}$ & 3 & 2 & 5 & 15 \\
\hline Demand & 75 & 20 & 50 & $145^{105}$ \\
\hline
\end{tabular}

\section{Case study-6:}

Table 17 considers an unbalanced TP which is solved by LS-ASM Method in Table 18 with total transportation cost $=10 \times 1+6 \times 60+4 \times 10+6 \times 10+3 \times 15+0 \times 40=515$.

\section{Real-life IFTP}

To realize the effectiveness of LS-ASM in real-life problem, a TIFTP from Kumar et al. [6] has been considered here in this section. The problem comprises of an umbrella company where the company manager wishes to transport umbrellas from three different factories to three different retail stores. The dataset for the transporation table is mentioned in Table 19 (see Table 20). 
Table 18 Optimal solution table using LS-ASM

\begin{tabular}{|c|c|c|c|c|}
\hline$s_{i}$ & $d_{1}$ & $d_{2}$ & $d_{3}$ & Supply \\
\hline$s_{1}$ & 5 & $1_{\mathbf{1 0}}$ & 7 & 10 \\
\hline$s_{2}$ & $6_{\mathbf{6 0}}$ & $4_{\mathbf{1 0}}$ & $6_{\mathbf{1 0}}$ & 80 \\
\hline$s_{3}$ & $3_{\mathbf{1 5}}$ & 2 & 5 & 15 \\
\hline Dummy & 0 & 0 & $0_{\mathbf{4 0}}$ & 40 \\
\hline Demand & 75 & 20 & 50 & 145 \\
\hline
\end{tabular}

Bold values in the respective mentioned tables signify equally optimal or better optimal solutions

Table 21 gives the optimal solution table for the reallife TIFTP solved by LS-ASM with total transportation cos $\mathrm{t}=9.75 \times 7.125+12.375 \times 4.625+17 \times 10.25+8.75 \times 8$. $75+14.75 \times 1.25=349.2656$.

\section{Results and discussion}

The techniques LS-ASM, ASM, VAM-MODI are being programmed in $\mathrm{C}++$. The code is compiled and executed on Intel(R) Core (TM) i5-7500 CPU (3.40 GHz) processor using Dev $\mathrm{C}++5.11$ compiler. The number of least-looping stepping-stone iterations required to reach optimality in each of the problems of Table 22 is 1 . The optimal transportation costs of all the methods are compared in Table 22. The bold letters represent the improved optimal solution reached by that particular method for the corresponding case study and real-life TIFTP.

The results of REDI [12] and Algorithm of [13] are directly taken from their respective papers. From Table 22, it is observed that the LS-ASM code executes successfully reaching the optimal solution. For the first two case studies, LS-ASM gives better optimal results than ASM [5] method, whereas for case study-3 and case study-4, it executes with same optimal results as other methods. A similar argument has been carried out for the case studies- 5 and 6 for
Table 20 Transportation of Table 19 after using the ranking function [6]

\begin{tabular}{lllll}
\hline & $D_{1}$ & $D_{2}$ & $D_{3}$ & Supply \\
\hline$S_{1}$ & 4.75 & 10.75 & 9.75 & 7.125 \\
$S_{2}$ & 5.125 & 12.375 & 17 & 14.875 \\
$S_{3}$ & 8.75 & 14.75 & 20.125 & 9.875 \\
Demand & 8.75 & 5.75 & 17.375 & \\
\hline
\end{tabular}

Table 21 Optimal solution table using LS-ASM

\begin{tabular}{lllll}
\hline & $D_{1}$ & $D_{2}$ & $D_{3}$ & Supply \\
\hline$S_{1}$ & 4.75 & 10.75 & $9.75_{7.125}$ & 7.125 \\
$S_{2}$ & 5.125 & $12.375_{4.625}$ & $17_{10.25}$ & 14.875 \\
$S_{3}$ & $8.75_{8.75}$ & $14.75_{1.25}$ & 20.125 & 9.875 \\
Demand & 8.75 & 5.75 & 17.375 & \\
\hline
\end{tabular}

Bold values in the respective mentioned tables signify equally optimal or better optimal solutions

unbalanced TPs and also for the real-life TIFTP considered in Section 3.4. Though the optimal solutions could not beat its near competitors namely, ASM and VAM-MODI, it has been able to obtain equally good results as that of others. Like balanced TPs, the LS-ASM consumes less time in case of unbalanced TPs too as compared to VAM-MODI over an average of 50 independent runs.

A fair comparison of these methods is done under the fixed platform and each algorithm is allowed to execute for 50 independent runs. The computational times of all the methods are compared in Table 23. Furthermore, the bold letters represent the least time required (in s.) for each individual case study and real-life TIFTP.

It is worth noting that LS-ASM consumes less computational time as compared to VAM-MODI method. Moreover, though LS-ASM takes bit more time than ASM for solving the problems in hand, but in return, LS-ASM provides better optimal solution than ASM as reported in Table 22 .

Table 19 A real-life TIFTP

\begin{tabular}{lllll}
\hline & $D_{1}$ & $D_{2}$ & $D_{3}$ & Supply \\
\hline$S_{1}$ & $(1,4,9 ; 0,4,12)$ & $(3,13,14 ; 2,13,15)$ & $(4,6,16 ; 1,6,33)$ & $(6,7,10 ; 2,7,11)$ \\
$S_{2}$ & $(4,5,7 ; 1,5,9)$ & $(5,10,15 ; 0,10,39)$ & $(7,16,24 ; 0,16,41)$ & $(6,15,23 ; 1,15,29)$ \\
$S_{3}$ & $(1,3,6 ; 0,3,10)$ & $(5,13,21 ; 5,13,35)$ & $(8,18,27 ; 6,18,48)$ & $(2,10,16 ; 0,10,21)$ \\
Demand & $(3,8,16 ; 0,8,19)$ & $(1,6,7 ; 0,6,14)$ & $(10,18,26 ; 3,18,28)$ & \\
\hline
\end{tabular}


Table 22 Total transportation costs

\begin{tabular}{llllll}
\hline $\begin{array}{l}\text { Transportation } \\
\text { problems (TPs) }\end{array}$ & \multicolumn{2}{l}{ Transportation cost } & & \\
\cline { 2 - 5 } & ASM & VAM-MODI & REDI [12] & Algorithm [13] & LS-ASM \\
\hline Balanced TP & & $\mathbf{4 1 0}$ & $*$ & $*$ & $\mathbf{4 1 0}$ \\
Case study-1 & 415 & $\mathbf{1 4 , 1 8 0}$ & $*$ & $*$ & $\mathbf{1 4 , 1 8 0}$ \\
Case study-2 & 14,380 & $\mathbf{1 1 8}$ & $*$ & $*$ & $\mathbf{1 1 8}$ \\
Case study-3 & $\mathbf{1 1 8}$ & $\mathbf{1 3 , 4 0 6 , 9 4 0 . 5}$ & $*$ & $*$ & $\mathbf{1 3 , 4 0 6 , 9 4 0 . 5}$ \\
Case study-4 & $\mathbf{1 3 , 4 0 6 , 9 4 0 . 5}$ & & & $\mathbf{1 7 , 8 7 5}$ \\
Unbalanced TP & & $\mathbf{1 7 , 8 7 5}$ & $\mathbf{1 7 , 8 7 5}$ & $*$ & $\mathbf{5 1 5}$ \\
Case study-5 & $\mathbf{1 7 , 8 7 5}$ & $\mathbf{5 1 5}$ & $*$ & $\mathbf{5 1 5}$ & Real-life TIFTP \\
Case study-6 & $\mathbf{5 1 5}$ & $*$ & $*$ & \\
Real-life TIFTP & $\mathbf{3 4 9 . 2 6 5 6}$ & $\mathbf{3 4 9 . 2 6 5 6}$ & $*$ &
\end{tabular}

Table 23 Average computational run times

\begin{tabular}{lllll}
\hline $\begin{array}{l}\text { Transportation } \\
\text { problems (TPs) }\end{array}$ & \multicolumn{4}{l}{ Avg. computational run times (in secs) } \\
\cline { 2 - 5 } & ASM & VAM-MODI & LS-ASM & Number of runs \\
\hline Balanced TP & & & 36.80 & 50 \\
Case study-1 & $\mathbf{3 1 . 4 1}$ & 39.66 & 40.63 & 50 \\
Case study-2 & $\mathbf{3 5 . 6 9}$ & 41.38 & 36.94 & 50 \\
Case study-3 & $\mathbf{2 9 . 6 7}$ & 40.57 & 78.74 & 50 \\
Case study-4 & $\mathbf{7 5 . 8 7}$ & 93.45 & & \\
Unbalanced TP & & & 19.54 & 50 \\
Case study-5 & $\mathbf{2 2 . 5 7}$ & 22.41 & 21.25 & 50 \\
Case study-6 & $\mathbf{2 3 . 2 5}$ & 24.55 & 29.76 & 50 \\
Real-life TIFTP & $\mathbf{2 6 . 8 1}$ & 34.57 & & \\
\hline
\end{tabular}

Bold values in the respective mentioned tables signify equally optimal or better optimal solutions

\section{Conclusion}

The manuscript proposes an improved stepping-stone approach (namely least-looping stepping-stone method), which has been synergized with a popular ASM approach. The newly framed approach thus established is named as LS-ASM. Unlike ASM, LS-ASM employs a least number of closed loops on non-basic cells. The proposed algorithm is allowed to solve both TPs (balanced and unbalanced) and a real-life TIFTP. Both the optimal solution obtained and the computational time consumed are being compared separately for LS-ASM with its near competitors like ASM and VAM-MODI. Since LS-ASM utilizes less time than the popular VAM-MODI in solving the TPs and TIFTP, it can be treated as an efficient method. On the other hand, LS-ASM provides equally competent with VAM-MODI. Thus, the efficiency of the LS-ASM over VAM-MODI is well established. Furthermore, though LS-ASM is a hybrid version of ASM, it is quite obvious that LS-ASM takes more executional time. However, due to its inherent characteristics of using the closed loops as minimum number of times as possible during simulation, the reachability of finding the optimal solution becomes faster. As a result, the performance of LS-ASM is either equally good or better than its original version, namely ASM. As a whole, it can be concluded that LS-ASM outperforms its near competitors in solving balanced, unbalanced, and TIFTP, as well. As a future scope of research, further improvement of LS-ASM can also be made with an effective modification in its existing algorithmic steps, so that the transportation cost will be further minimized and/or the computational time will be further reduced. Also, it can be efficiently applied on several other unbalanced real-world TPs and IFTPs.

\section{Declarations}

Conflict of interest statement On behalf of all authors, the corresponding author states that there is no conflict of interest.

Open Access This article is licensed under a Creative Commons Attribution 4.0 International License, which permits use, sharing, adaptation, distribution and reproduction in any medium or format, as long as you give appropriate credit to the original author(s) and the source, provide a link to the Creative Commons licence, and indicate if changes were made. The images or other third party material in this article are included in the article's Creative Commons licence, unless indicated otherwise in a credit line to the material. If material is not included in the article's Creative Commons licence and your intended use is not permitted by statutory regulation or exceeds the permitted use, you will need to obtain permission directly from the copyright holder. To view a copy of this licence, visit http://creativecommons.org/licenses/by/4.0/.

\section{References}

1. Hitchcock FL (1941) The distribution of a product from several sources to numerous localities. J Math Phys 20:224-230

2. Zadeh LA (1965) Fuzzy sets. Inf Control 8:338-353 
3. Bellman R, Zadeh LA (1970) Decision making in Fuzzy environment. Manag Sci 17(B):141-164

4. Atanassov KT (1986) Intuitionistic fuzzy sets. Fuzzy Sets Syst 20:87-96

5. Quddoos A, Javaid S, Khalid MM (2012) A new method for finding an optimal solution for transportation problems. Int J Comput Sci Eng (IJCSE) 4(7):1271-1274

6. Kumar PS, Hussain RJ (2016) Computationally simple approach for solving fully intuitionistic fuzzy real life transportation problems. Int J Syst Assur Eng Manag 7(Suppl. 1):90-101

7. Singh SK, Yadav SP (2014) A new approach for solving intuitionistic fuzzy transportation problem of type-2. Springer Science + Business Media, New York

8. Ahmed MM, Khan AR, Sharif Uddin MD, Ahmed F (2016) A new approach to solve transportation problems. Open J Optim 5:22-30

9. Murthy PR (2007) Operation research. New Age International (P) Limited, New Delhi

10. Antony RJP, Savarimuthu SJ, Pathinathan T (2014) Method for solving the transportation problem using triangular intuitionistic fuzzy number. Int J Comput Algorithm 3:590-605

11. Chhibber D, Bisht DCS, Srivastava PK (2019) Ranking approach based on incenter in triangle of centroids to solve type-1 and type-2 fuzzy transportation problem. In: AIP Conference Proceedings 2061: 020022

12. Aramuthakannan S, Kandasamy PR (2016) Application of revised distribution method for finding optimal solution of unbalanced transportation problem. PARIPEX-Indian J Res 5(1):39-42

13. Halawa MIA, Maatuk AM, Idrees HS, Ali EM (2016) An optimal solution for transportation problem using computing modelling. In: International Conference on Engineering and MIS (ICEMIS), p 1-5. IEEE

14. Roy GC, Hossain E (2015) Operation research. Titus Publication

15. Hasan MK (2012) Direct methods for finding optimal solution of a transportation problem are not always reliable. Int Ref J Eng Sci (IRJES) 1(2):46-52

16. Shakeel AQ, Khalid MM (2016) A revised version of ASM method for solving transportation problem. Int J Agric Stat Sci 12(Supplement 1):267-272
17. Murugesan R (2019) A note on: direct methods for finding optimal solution of a transportation problem are not always reliable. Int Ref J Eng Sci (IRJES) 8(3):39-48

18. Murugesan R, Esakkiammal T (2019) Revised version of ASM method-the best one for finding an IBFS for transportation problems. In: International Conference on recent advances in pure and applied mathematics (ICRAPAM-2019)

19. Murugesan R, Esakkiammal T (2020) Revised version of ASM method-the best one for finding an IBFS for transportation problems. Adv Math Sci J 8(3):493-510

20. Murugesan R, Esakkiammal T (2019) A comparative study on ASM method with harmonic mean approach in transportation problems. In: International Conference on mathematical analysis and computing (ICMAC-2019), Organized by the Department of Mathematics, SSN College of Engineering, Kalavakkam, Chennai, Tamil Nadu, India

21. Murugesan R, Esakkiammal T (2019) A comparative study on ASM method with MDMA method in balanced transportation problems. In: International Conference on operations research and decision systems (ICORDS-2019), organized by the Indian Institute of Management, Visakhapatnam, Andhra Bank School of Business, Andhra University Campus, Visakhapatnam

22. Murugesan R, Esakkiammal T (2019) A comparative study on ASM method with MDMA method in transportation problems. Int J Comput Appl Math (IJCAM) (accepted)

23. Murugesan R, Esakkiammal T (2020) A comparative study on ASM Method with VAM and ATM methods in transportation problems. In: International Conference on innovations in graphs and its alliances in digital era (ICIGA-2020), Organized by Department of Mathematics, University of Kerala, Kariavattom, Thiruvananthapuram, Kerala, p 27

Publisher's Note Springer Nature remains neutral with regard to jurisdictional claims in published maps and institutional affiliations. 